\title{
Magnetohydrodynamic Calculations of Toroidal Fusion Reactor to Ensure Stable Control
}

\author{
Aybaba Hançerlioğullari, Asli Kurnaz, Yosef G. Ali Madee \\ Department of Physics, Faculty of Arts and Sciences, Kastamonu University, Kastamonu, Turkey \\ Email: aybaba@kastamonu.edu.tr, youseff77@yahoo.com, akurnaz@kastamonu.edu.tr
}

Received 31 May 2016; accepted 11 July 2016; published 14 July 2016

Copyright (C) 2016 by authors and Scientific Research Publishing Inc.

This work is licensed under the Creative Commons Attribution International License (CC BY).

http://creativecommons.org/licenses/by/4.0/

(c) () Open Access

\section{Abstract}

The development of magnetic configurations to confine the stability fluid plasmas for fusion energy is a challenge that is a mixture of basic fusion engineering and invention. In order to keep the fusion reactions in the plasma to be continuing in the fusion reactors, the speed of tritium breeding (TBR) should be kept above a certain value. At the Apex fusion reactor, a fast flowing thin liquid wall has replaced the solid first wall concept of the traditional reactors. Behind the fast flowing thin liquid wall, a slower and thicker second liquid wall (coat) is present. Monte Carlo Random method (MCRS) is the general name for the solution of experimental and statistical problems with a random approach. This method is dependent upon the theory of probability. In the present work, Mhd impacts are investigated quite unimportant for Flibe salt solutions. In this study, the fissile fuel production calculations are done for a neutron wall load of $10 \mathrm{MW} / \mathrm{m}^{2}$ fissile fuel production rates of ${ }^{238} \mathrm{U}(\mathrm{n}, \gamma)^{239} \mathrm{Pu}$ and ${ }^{232} \mathrm{Th}(\mathrm{n}, \gamma)^{233} \mathrm{U}$ increases almost linearly with increased heavy metal content.

\section{Keywords}

Fusion Reactor, Monte Carlo, Magnetohydrodynamic, Tritium Breeding (TBR)

\section{Introduction}

Apex fusion reactor has an advantage of high neutron wall load capability due to its high beta. Controlled nuclear fusion can be a very important energy for future. At the Apex fusion reactor, a fast flowing thin liquid wall has replaced the solid first wall concept of the traditional reactors. In this study, Flibe salt solutions are used in the liquid wall of the reactor. The results obtained for both salt solutions are compared. At the construction of 
the liquid wall, it is possible to use liquid metals but it might be necessary to use electric isolators to manage magnetic-hydrodynamic drafts at the liquid metal flows, and as this will affect the plasma stability which maintains vital importance for fusion reactors, it is an important disadvantage for the usage of liquid metals. The application of the method to a problem is based on the idea of an approximate calculation of the parameter that is aimed to be calculated, using the random numbers of the problem by considering the results of this simulation [1]-[3]. A random number generator is a critical component in modern cryptographic communication systems, statistical simulation systems and scientific area incorporating Monte Carlo methods [4]. The general look of the linear equation that will form the random number series is as follows: $x_{\mathrm{n}+1}=\left(a x_{\mathrm{n}}+b\right)$ mode $m$. Here mode means modular arithmetic procedure. The first element of the series $x_{0}$ is called the seed [5]-[7]. Constants $a$ and $b$ are chosen so that the elements of the series will be distributed anarchic and orderly. The same research company has used the following famous Random algorithm.

$(a=69,069, b=0): x_{n+1}=\left(69,069 x_{n}\right) \bmod 2^{31}-1 . x_{n+1}=\left(16,807 x_{n}\right)$ mod $2^{31}$. However, with appropriate algorithms, a computer may be forced to generate Random numbers chosen from an orderly scatter and takes real numbers between $(0-1)$. Especially in nuclear field, using the nuclear transport codes, by the aid of MCNP (Monte Carlo n-particle transport code), physical and dynamical events are resulted by repeated utilization of the random numbers.

\section{Method}

\subsection{Control of Plasma Position}

Plasma is a collection of charged particles which is macroscopically neutral over a volume that is small compared to its dimensions. Nuclear fusion research has been most actively pursued by the use of hot plasma. In principle, the motion of each particle can be determined from Newton's second Law and the electrostatic force that each particle exerts on all other particles. The magnetic plasma method must be heated and confined in order to utilize the nuclear fusion energy released from the plasma with a high temperature. The plasma consists of charged particles. Charged particles cannot cross magnetic field lines, but rotate around the lines. There is the outline of the idea of magnetic field lines in which to confine the plasma. In Figure 1 as shown, elliptic torus of fusion design cross-section, the particle drifts in the presence of a purely torodial magnetic field. The B magnetic gradient and curvature drifts produce a charge separation, which, in turn, produces an electric field with an associated outward drift for both ions and electrons and loss of confinement. In the geometry, a torus is a surface of motion generated by revolving a circle in 3D space.

When the magnetic field changes slowly, the magnetic moment is conserve. Therefore, if $B$ is increased, $\mathrm{mv}^{2} / 2=\mu \mathrm{B}$ is also increased and the particles are heated. This kind of heating is called adiabatic heating. This solution shows that the guiding center motion consists of usual ExB drift and the new drift along $E$. Plasmas can be described as magnetohyrodynamic two fluids of ions and electrons with mass densities $\rho_{m i}, \rho_{\mathrm{me}}$, charge density $\rho$, current density $j$, flow velocities $V i$, Ve and pressures $P i, P e$.

The number of ions $\mathrm{dNi}$ in a small phase space volume,

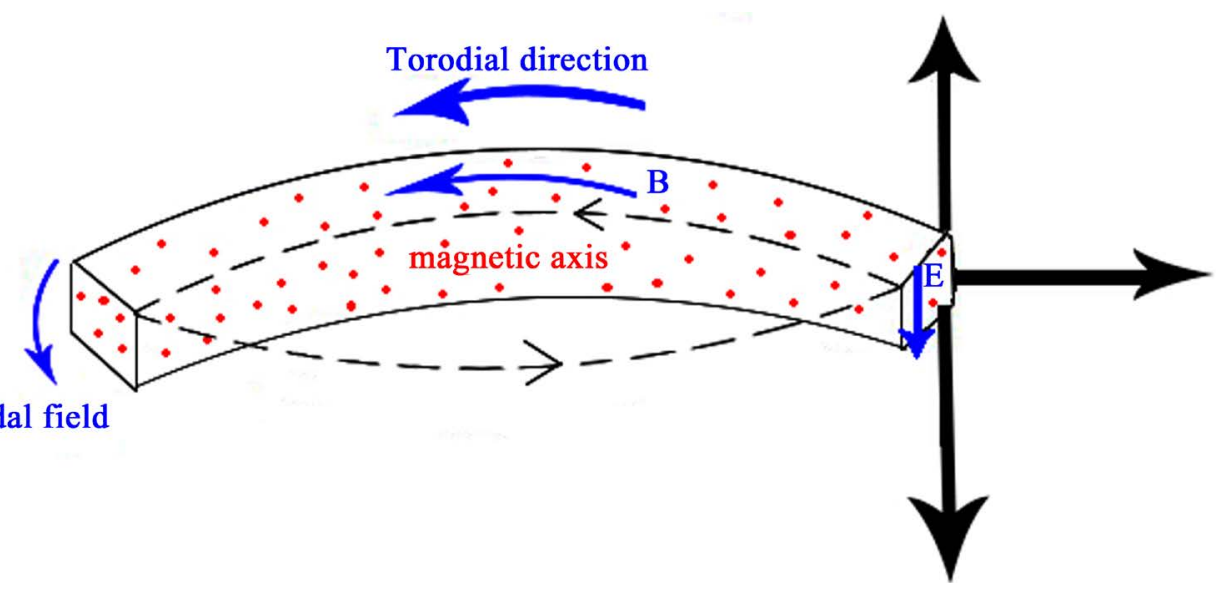

Figure 1. Elliptic torus model of apex fusion design. 


$$
\mathrm{d} N i=\mathrm{d} x \mathrm{~d} y \mathrm{~d} z \mathrm{~d} V x \mathrm{~d} V y \mathrm{~d} V z
$$

Magnetohydrodynamic (MHD) equations are the following;

$$
\begin{aligned}
& \frac{\partial n e}{\partial t}+\nabla \cdot(n e V e)=0 \\
& \frac{\partial n e}{\partial t}+\nabla \cdot(n i V i)=0 .
\end{aligned}
$$

\subsection{The Utilization of the MCSR for Reactor Design}

In the Monte Carlo Method, random numbers are generated for each element and for each random number, an examination is conducted in order to determine if the system obtained or not. There are practical manual methods for the generation of the Random numbers as well as a number of mathematical methods. According to the status of the analyzed system, there are practical methods like tossing dices, heads or tails or bingo, as will be understood from the name Monte Carlo. MCNP code includes Monte Carlo simulation and physics and mathematics subjects that have nuclear characteristics including certain subjects. During particle transport, neutrons, photons and electrons are used. MCNP in fact is a code that has been generalized by the Monte Carlo group in los-alamos labs for theoretic physics [5] [6] [8] [9]. MCNP possesses a general block that contains approximately 40,000 lines of Fortran and 1000 lines C source coder that includes interpretations and a general block that applies the program Monte Carlo Simulation is used in the simulation of some physical and mathematical systems. Generally, it is used for analyzing the effect of the random variables on the system and operates mostly with an algorithm that generates random numbers for the variables. The variables for which Monte Carlo simulation is to be conducted may be generated in a way that will suit a specific statistical distribution [4] [8]-[11]. The opposite of complementary probability distribution function (CDF) is used. The random numbers that are uniformly generated by the computer at a certain interval $(0-1)$ may easily be transformed to any kind of distribution using the opposite of the

$$
\begin{aligned}
& { }^{238} \mathrm{U}+n \rightarrow{ }^{239} \mathrm{U} \stackrel{\beta^{-}}{\longrightarrow}{ }^{239} \mathrm{~Np} \stackrel{\beta^{-}}{\longrightarrow}{ }^{239} \mathrm{Pu} \\
& { }^{232} \mathrm{Th}+n \rightarrow{ }^{233} \mathrm{Th} \stackrel{\beta^{-}}{\longrightarrow} \mathrm{Pa} \stackrel{\beta^{-}}{\longrightarrow}{ }^{233} \mathrm{U} .
\end{aligned}
$$

The design of $3 \mathrm{~d}$ torus of fusion reactor the plasma and the blanket lithium around the plasma and the structural element that contains $U$ and $T h$ are shown in Figure 2.

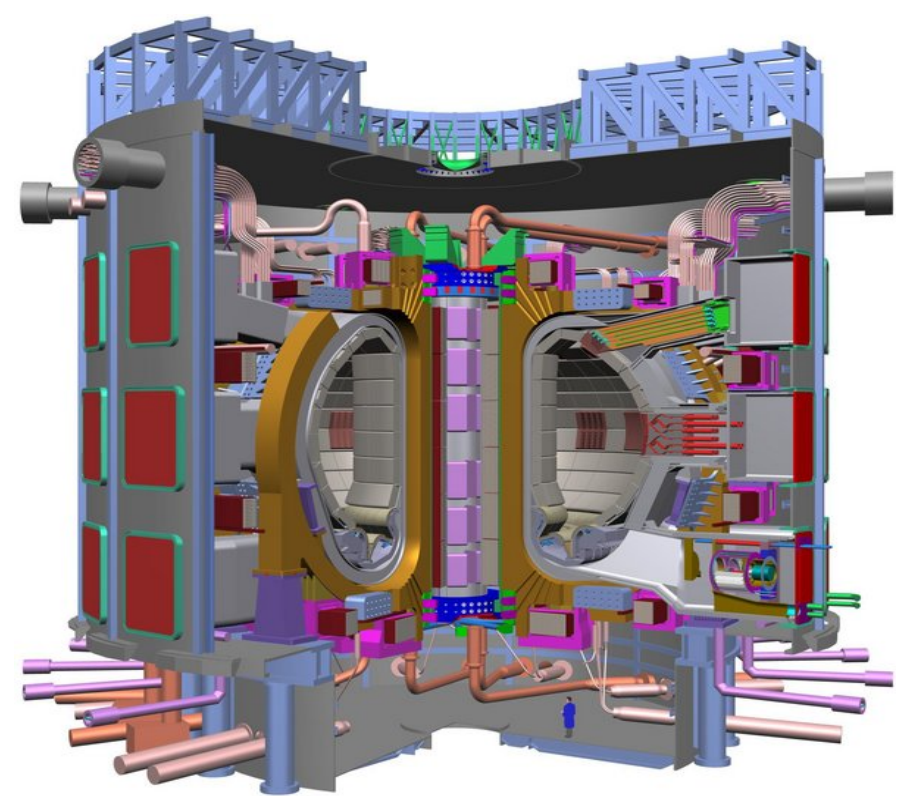

Figure 2. 3D torus geometry of the apex reactor for flibe liquid flow [12] [13]. 
The neutrons that are radiated as a result of the fusion reactions that occur at the plasma are captured at the blanket by uranium, thorium and the production of fissile fuel is provided. It is not possible to combust $\mathrm{U}^{238}$ in most reactors because the fusion impact incision is very low and the activation energy is too high. Th ${ }^{232}$ also is not an appropriate material for combustion [12] [14] [15]. Monte Carlo Random Simulation (MCRS), models the probable systems based on randoms. It is possible to calculate the integrals with the Monte Carlo Method through phase transitions [5] [6] [8]-[10].

$f(x)$ function at the $[\mathrm{a}, \mathrm{b}]$ interval is considered : $I=\int_{a}^{b} f(x) \mathrm{d} x \quad x=\left(x^{1}, x^{2}, \cdots, x^{d}\right)$ din order to show the size of the integral, quadratic total demonstration of such an integral shall be,

$I=\int_{R} f(x) \mathrm{d} x=\Delta x^{d} \sum_{i_{1}=1}^{N} \sum_{i_{2=1}}^{N} \ldots \sum_{i_{3}=1}^{N} f\left(x_{i_{1}}^{1}, \cdots, x_{i_{d}}^{d}\right)$. According to the Average value theorem, the integral of a $f(x)$ function at $[a, b]$ interval is equal to the area of the rectangle which is drawn at the average value of the function as seen on Figure 2; $I=(b-a)\langle f\rangle$ then $\langle f\rangle$ average value is multiplied by $(\mathrm{b}-\mathrm{a})$ and the integral is calculated.

If the arithmetic average of $\mathrm{N}$ number of dots that are chosen randomly at the, [a, b] interval of the function is calculated, it will be: $\langle f\rangle=\frac{1}{N} \sum_{i=1}^{N} f\left(x_{i}\right)$ So, reaches the Monte Carlo integration formula: $I=\frac{b-a}{N} \sum_{i=1}^{N} f\left(x_{i}\right), x_{1}, x_{2}, \cdots, x_{N}$ the dots have not been arranged with equal intervals, they are chosen completely randomly. As number $N$ increases, the numeric calculation gets closer to the value of the $I$ integral. But this approach is not as fast as expected. This slow approach is a general characteristic of the random methods. Here, according to the result that is provided without proof, the margin of error decreases slowly as $1 / \sqrt{N} \quad[6][11][16]$.

\section{Results and Discussion}

The apex fusion reactor used in the study has designed by using MCNP-4b (Endf/b-iv-vi) computer code and the Monte-Carlo technique, as 3-D torus [8]-[10] [16]. The dimensions for the apex reactor were taken from the aries-rs reactor design which has made in the framework of apex studies. A cross-sectional view of apex designed by using MCNP-4b computer code is shown in Figure 4. The inner region consists of plasma and vacuum. The first liquid wall is enclosed by the liquid blanket and then ferritic steel; these are further enclosed by a shield a stainless steel and ferritic steel zone. In this model, the radius of torus is $552 \mathrm{~cm}$ and minor radius starting from inner surface of first wall is $143 \mathrm{~cm}$. By preserving a self sufficient tritium breeding ratio (TBR > 1.12) for a mole fraction up to $12 \%$ of UF4, the modified APEX reactor can produce up to $\sim 3000 \mathrm{~kg}$ of 233 U/year or $\sim 5000 \mathrm{~kg}$ of $239 \mathrm{Pu} /$ year, assuming the same baseline fusion power production of $4000 \mathrm{MWth}$, as in the original APEX concept 50\% UF4 in the coolant, the total energy output will increase to 6560 MWth or 9440 MWth, respectively. For a plant operation period of 30 full power years, the atomic displacement and helium production rates remain well below the presumable limits [17]-[19].

The height of torus starting from center of first wall is $250 \mathrm{~cm}$. The radius and thicknesses in one dimension are shown in detail in Figure 3 [10] [11] [16] [20].

\subsection{Neutron Tracking}

Neutron tracking refers to the process of simulating the movement of a single neutron through the different material regions of the geometry. The shortest length of path that the neutron makes between the points of interaction is called a track. The track can also be cut short by a material boundary surface located between the two points. The entire set of tracks made from the initial emission to the final absorption or escape is called a neutron history.

The number of neutron histories is hence equal to the total number of emitted source neutrons. The basis of the simulation process is the sampling of the free path length between two collision points. Figure 4 shows the estimation of Monte Carlo random errors of number of neutron histories. 


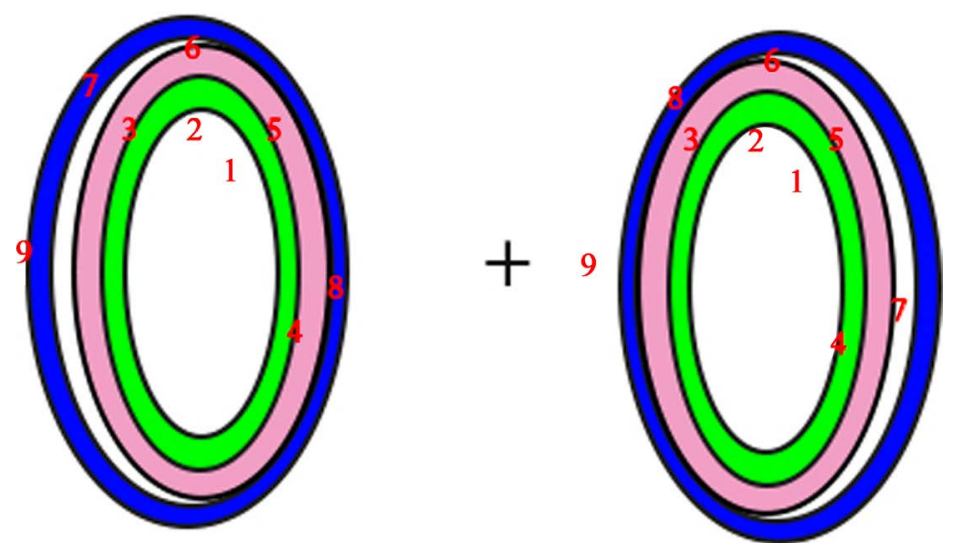

Figure 3. Inside-out cell and surface cross section of Apex fusion reactor model designed in MCNP-4b [10] [16].

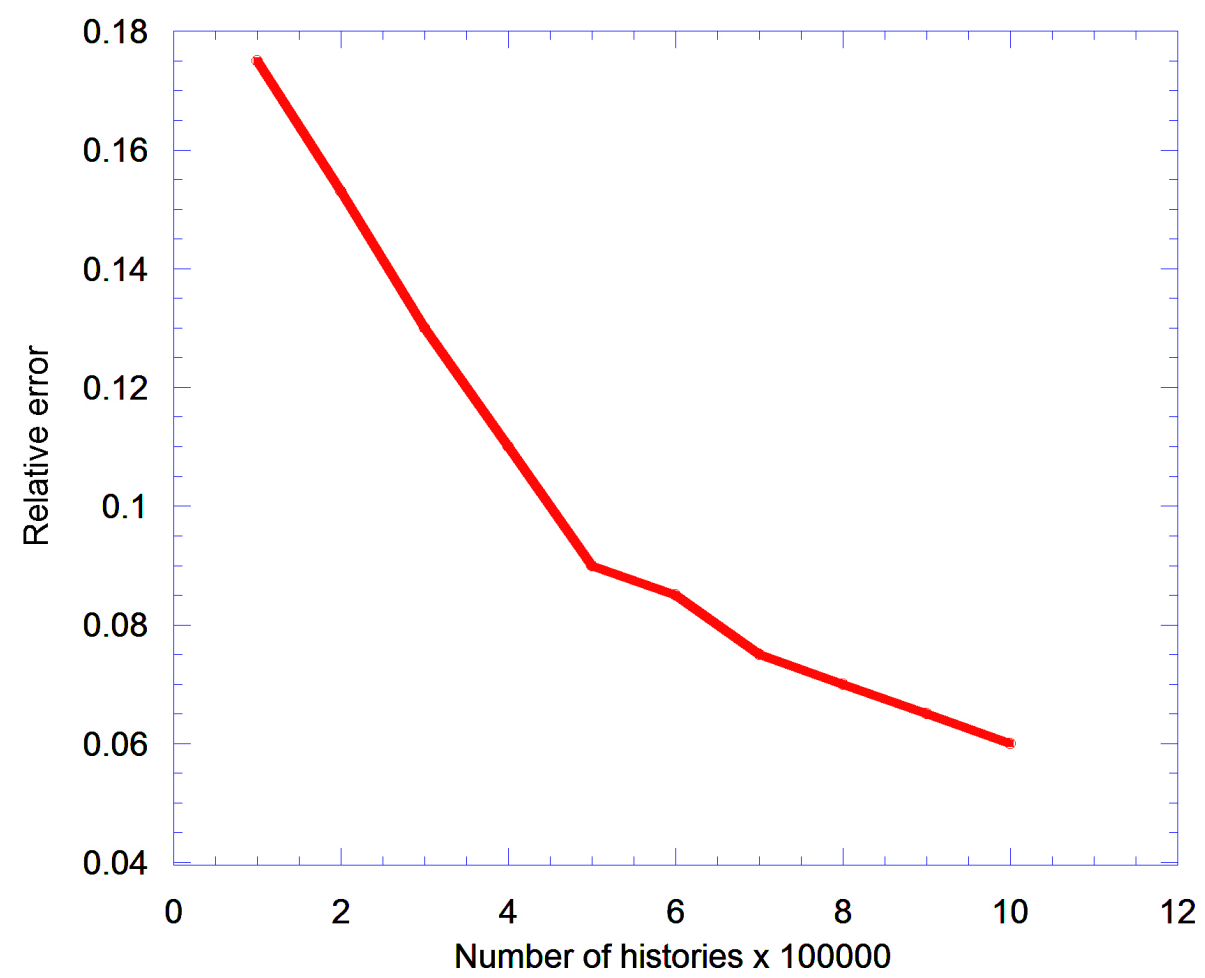

Figure 4. Estimation of Monte Carlo errors of number of neutron histories.

\subsection{Tritium Breeding and Fissile Productions}

A commercial fusion reactor must have a tritium breeding ratio of (TBR) $>1.05$ to be self sustaining. Figure 5 shows the variation of total fissile material production speed for Flibe due to the ratio of UF4 and ThF4. As expected, the TBR decreases with increased heavy metal content. Tritium self sufficiency has been maintained in the range of molten salt mixtures (Flibe with up to 10\% - 50\% UF4) are shown results in Table 1 . These molten salt mixtures would make the highest level of fissile breeding possible. Uranium has a higher neutron multiplication than thorium. On the other hand, the excess tritium gain for external fusion reactors is reduced significantly with the introduction of heavy metal into the coolant. When the fast and slow neutrons interaction with the lithium blanket, $\mathrm{Li}$ and $\mathrm{T}$ are produced as shown [15] [21] [22];

$$
\begin{aligned}
& \mathrm{n}(\text { slow })+{ }^{6} \mathrm{Li} \rightarrow \mathrm{T}+{ }^{4} \mathrm{He}+4.8 \mathrm{MeV} \\
& \mathrm{n}(\text { fast })+{ }^{7} \mathrm{Li} \rightarrow{ }^{4} \mathrm{He}+n^{\prime}-2.5 \mathrm{MeV}
\end{aligned}
$$


Table 1. Modeling of the apex for nuclear problems by simulating.

\begin{tabular}{ccccccc}
\hline TYPES & Type of Cooling & $\mathrm{TBR}=\mathrm{T}_{6}+\mathrm{T}_{7}$ & $\mathrm{M}$ & $\Sigma_{f}$ & $v \Sigma_{f}$ & $\Sigma_{\gamma}$ \\
\hline 1 & Natural Flibe (100\%) & 1.3274 & 1.7394 & 0.00000 & 0.00000 & 0.00000 \\
2 & $10 \%$ Uf4 + 90\% Flibe & 1.2728 & 1.8434 & 0.01013 & 0.03618 & 0.08788 \\
3 & 20\% Uf4 + 80\% Flibe & 1.2584 & 1.9598 & 0.02025 & 0.07220 & 0.13370 \\
4 & 30\% Uf4 + 70\% Flibe & 1.2390 & 2.0746 & 0.03037 & 0.10816 & 0.17517 \\
5 & 40\% Uf4 + 60\% Flibe & 1.2031 & 2.1934 & 0.04055 & 0.13886 & 0.21642 \\
6 & 50\% Uf4 + 50\% Flibe & 1.207934 & 2.3072 & 0.05066 & 0.18005 & 0.25340 \\
\hline
\end{tabular}

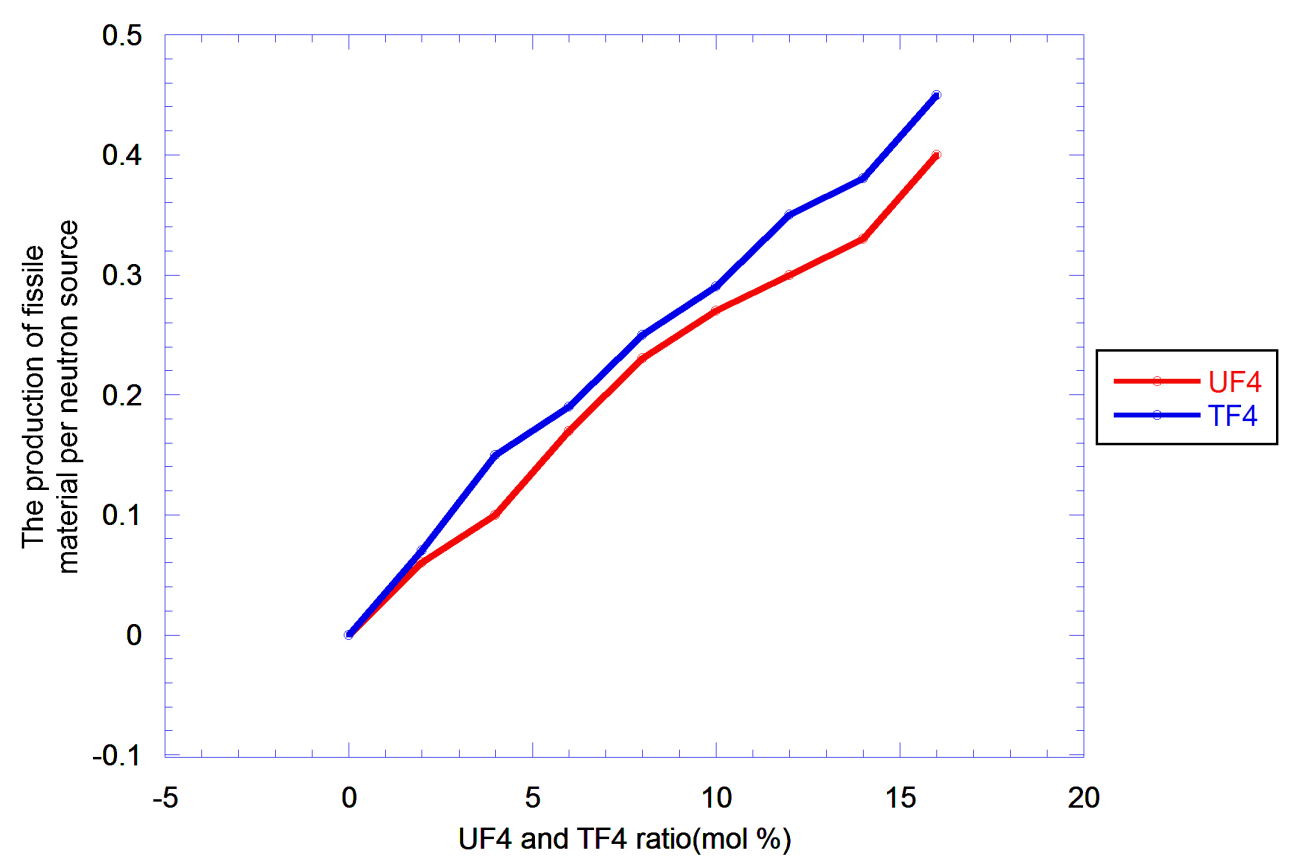

Figure 5. Total fissile material production speed for Flibe due to the ratio of UF4 and ThF4.

where, $\mathrm{M}$ is the energy multiplication factor, $\Sigma_{\gamma}$ is fissile fuel production ratio, $v \Sigma_{f}$ is ratio of total fission and $v$ is total number of fusion neutron. In Figure 5 as shown fissile fuel breeding are done fissile fuel breeding rates of ${ }^{238} \mathrm{U}(\mathrm{n}, \gamma)^{239} \mathrm{Pu}$ and ${ }^{232} \mathrm{Th}(\mathrm{n}, \gamma)^{233} \mathrm{U}$ increase almost linearly with increased heavy metal content.

\section{Conclusion}

In fusion and hybrid reactors, it is necessary to provide the Tbr $>1.05$ terms in order to maintain the continuity of the operation of the reactors. At the situation when there are no additions made to the blanket, the value $\mathrm{Tbr}=$ 1.20 for flibe is observed. The most important feature of hybrid reactor is that the first wall surrounding the plasma is liquid. For the D-T fusion driver, the energy multiplication factor, M, is defined in Figure 5, as the ratio of the total energy releases in the blanket to the incident fusion neutron energy [12]-[15]. For uranium, it also has been calculated that UF4 added hybrid reactor with UF4 ratios between 10\% - 50\% working with a wall load of $10 \mathrm{MW} / \mathrm{m}^{2}$ also works about twice as powerful as the original APEX Fusion reactor. Besides, the fissile fuel production provided at the reactor is also of great importance.

\section{References}

[1] Hançerlioğullari, A. (2006) Monte Carlo Simulation Method and MCNP Code System. Kastamonu Education Journal, 14, 545-556.

[2] Sobol Ilya, M. (1994) A Primer for the Monte Carlo Method. CRC Press, London.

[3] Rod Blais, J.A. and Zhang, Z. (2011) Exploring Pseudo and Chaotic Random Monte Carlo Simulations. Computer \& 
Geosciences, 37, 928-934. http://dx.doi.org/10.1016/j.cageo.2011.01.009

[4] Behnia, S., Akhavan, A., Akhshani, A. and Samsudin, A. (2011) A Novel Dynamic Model of Pseudo Random Number Generator. Journal of Computational and Applied Mathematics, 235, 3455-3463. http://dx.doi.org/10.1016/j.cam.2011.02.006

[5] Binder, K. (1997) Applications of Monte Carlo Methods to Statistical Physics. Reports on Progress in Physics, 60, 487-559

[6] Carter Jr., E.F. (1999) Random Walks, Markov Chains and the Monte Carlo Method, Monte Carlo Integration. Taygeta Scientific Inc. https://www.taygeta.com/rwalks/node5.html

[7] Pevey, R.E. (1998) Monte Carlo Course Notes. Department of Nuclear Engineering, the University of Tennessee, Knoxville. Spring Semester. http://web.utk.edu/ rpevey/

[8] Foster, D. and Artur, G. (1982) Average Neutronic Properties of "Prompt” Fission Poducts. Los Alamos National Laboraty Report la-9168-ms, February.

[9] Johston, R.A. (1963) General Monte Carlo Neutronics Code. Lams-2856, Los Alamos.

[10] Briesmeister, J. (1993) RSIC Computer Code Collection MCNP 4a, Monte Carlo N-Particle Transport Code System. Los Alamos National Laboratory, New Mexico.

[11] Hançerlioğullari, A. (2011) Determining of Energy Multiplication in the Apex Hybrid Reactor by Using ThF4 and UF4 Heavy Metals Salts. International Journal of Energy Research, 36, 1375-1382.

[12] Abdou, M.A. (1999) Exploring Novel High Power Density Concepts for Attractive Fusion System. Fusion Engineering \& Design, 4, 67-145.

[13] Moir, R.W. (1997) Liquid First Walls for Magnetic Fusion Energy Configurations. Nuclear Fusion, 37, 557. http://dx.doi.org/10.1088/0029-5515/37/4/I13

[14] Abdou, M.A. and The Apex Team (1999) Exploring Novel High Power Density Concepts for Attractive Fusion Systems. Fusion Engineering and Design, 45, 145-167. http://dx.doi.org/10.1016/S0920-3796(99)00018-6

[15] Abdou, M.A., Ying, A. and Morley, N. and The Apex Team (2001) On the Exploration of Innovative Concepts for Fusion Chamber Technology. Fusion Engineering and Design, 54, 181-247. http://dx.doi.org/10.1016/S0920-3796(00)00433-6

[16] Sarer, B., Hançerlioğullari, A. and Savruk, N. (2005) A Design for Apex Hybrid Reactor Model by Using Monte Carlo Method. G.U. Journal of Science, 18, 17-25.

[17] Şahin, H.M. (2007) Monte Carlo Calculation of Radiation Damage in First Wall of an Experimental Hybrid Reactor. Annals of Nuclear Energy, 34, 861-870.

[18] Doucet, A., Johansen, M.A. and Tadic, B.V. (2010) On Solving Integral Equations Using Markov Chain Monte Carlo Methods. Applied Mathematics and Computation, 216, 2869-2880. http://dx.doi.org/10.1016/j.amc.2010.03.138

[19] Beyli, M.U. and Acir, A. (2007) Utilization of Thorium in a High Power Density Hybrid Reactor with İnnovative Coolants. Energy Conversion and Management, 48, 576-582. http://dx.doi.org/10.1016/j.enconman.2006.06.007

[20] Maziar, N., Matthew, C.W. and Needs, J.R. (2003) Quantum Monte Carlo Studies of Density Functional Theory. Mathematics and Computers in Simulation, 62, 463-470.

[21] Mahdavi, M. and Asadi, E. (2013) Estimates of Tritium Produced Ratio in the Blancet of Fusion Reactors. Open Journal of Microphysics, 3, 8-11. http://dx.doi.org/10.4236/ojm.2013.31002

[22] Hançerlioğullari, A. (2014) Thermodynamics Properties of Molten Salt Technology Assessment for New Generation Fusion Reactors. Journal of Fusion Energy, 33, 5. 


\section{Submit or recommend next manuscript to SCIRP and we will provide best service for you:}

Accepting pre-submission inquiries through Email, Facebook, LinkedIn, Twitter, etc.

A wide selection of journals (inclusive of 9 subjects, more than 200 journals)

Providing 24-hour high-quality service

User-friendly online submission system

Fair and swift peer-review system

Efficient typesetting and proofreading procedure

Display of the result of downloads and visits, as well as the number of cited articles

Maximum dissemination of your research work

Submit your manuscript at: http://papersubmission.scirp.org/ 